\title{
A novel recombinant BCG expressing pro-apoptotic protein BAX enhances Th1 protective immune responses in mice
}

\author{
Guanghua Li ${ }^{\mathrm{a}}$, Guoyuan Liu ${ }^{\mathrm{b}}, \mathrm{Na}$ Song ${ }^{\mathrm{a}}$, Cong Kong ${ }^{\mathrm{a}}$, Qi Huang ${ }^{\mathrm{a}}$, \\ Haibo $\mathrm{Su}^{\mathrm{a}}$, Aixiao $\mathrm{Bi}^{\mathrm{c}}$, Liulin $\mathrm{Luo}^{\mathrm{c}}$, Lin $\mathrm{Zhu}^{\mathrm{a}}$, Ying $\mathrm{Xu}^{\mathrm{a}, *}$ and Honghai Wang ${ }^{\mathrm{a}, *}$
}

a. State Key Laboratory of Genetic Engineering; Institute of Genetics; School of Life Science, Fudan University; 2005 Songhu Road, 200438, Shanghai, People's Republic of China

b. Department of Pathology, School of Basic Medical Sciences, Fudan University; Shanghai, People's Republic of China

c. Shanghai Pulmonary Hospital, Tongji University School of Medicine, Shanghai, People's Republic of China

*Correspondence authors: Honghai Wang and Ying Xu; E-mail addresses: hhwang@fudan.edu.cn , yingxu2520@fudan.edu.cn; Phone number: +86 2151630588 and +86 2151630587

\section{ABSTRACT}

One-third of the world's population is infected with Mycobacterium tuberculosis (MTB). The protective efficacy of bacille Calmette Guérin (BCG) vaccine against tuberculosis (TB) in adults is highly controversial even though the BCG vaccine has been available for more than 90 years. Because BCG is effective against infantile tuberculosis meningitis and miliary tuberculosis in young children and provides cost-effective prevention from tuberculosis for developing countries, it would be desirable to modify the existing BCG vaccine to provide more comprehensive protection. In our study, we constructed a novel recombinant BCG strain expressing pro-apoptotic BAX (rBCG::BAX) and demonstrated that it significantly induced the apoptosis of macrophages infected with rBCG::BAX both in vitro and in vivo. In addition, it significantly enhanced 
Ag85B-specific IFN- $\gamma$ enzyme-linked immunospot responses, IFN- $\gamma$ secretion, IL-2 secretion and the ratio of Ag85B-specific $\mathrm{IgG} 2 \mathrm{~b} / \mathrm{IgG1}$, and it significantly decreased Ag85B-specific IL-4. Furthermore, it presumably facilitated antigen presentation by inducing a significant up-regulation in the expression of MHC-II and B7.1 (CD80) co-stimulatory molecules on macrophages. In conclusion, these results suggest that the rBCG::BAX strain elicited predominantly a Th1 protective immune responses and might be a potential tuberculosis vaccine candidate for further study.

Keywords: tuberculosis; vaccine; recombinant BCG; apoptosis; BAX

\section{Introduction}

One-third of the world's population is infected with Mycobacterium tuberculosis (MTB). According to the WHO 2014 global tuberculosis report, an estimated 9.0 million people developed tuberculosis (TB), and 1.5 million people died from TB in 2013 (Zumla et al., 2015)although the bacille Calmette Guérin (BCG) vaccine has been available for more than 90 years. In 1974, BCG vaccination against tuberculosis was included in the EPI (the Expanded Programme Immunization) and now covers $80 \%$ of all infants around the globe (2013; Hatherill, 2011). With more than 4 billion vaccinations (2013), BCG is effective against infantile tuberculosis meningitis and miliary tuberculosis (Trunz et al., 2006), but it fails to provide reliable protection against pulmonary tuberculosis in adults (Colditz et al., 1994; Svenson et al., 2010). Furthermore, tuberculosis drug treatment is lengthy and costly, and reduced compliance has caused an increase in 
multidrug-resistant strains. To reduce the immense burden of tuberculosis, both new vaccines and vaccination strategies are urgently needed.

Currently, TB vaccines are divided into three subtypes, which are either preventive or therapeutic. The preventive strategies consist of priming vaccines to replace BCG and subunit vaccines as boosters for BCG prime (Andersen and Kaufmann, 2014). One of the therapeutic candidates that has reached clinical trials is a killed whole mycobacteria vaccine. BCG-replacement vaccines are based either on improvement of BCG live vaccines, known as recombinant BCG (rBCG), or on genetic attenuation of Mycobacterium tuberculosis through the deletion of virulence genes, such as MTBVAC01 lacking the phoP and the fadD26 gene loci (Kaufmann and Gengenbacher, 2012). Because BCG confers significant protection in adolescents and provides cost-effective prevention from serious forms of disseminated TB in developing countries, it would be desirable to modify the current BCG vaccine to provide more comprehensive protection. Currently, the rBCG $\Delta$ UreC::Hly (VPM1002) vaccine has successfully passed a phase I clinical safety trial (Grode et al., 2013) and has completed a phase IIa trial in infants in South Africa (Kaufmann et al., 2014). VPM1002 is a recombinant BCG strain that expresses listeriolysin (Hly) derived from Listeria monocytogenes to perforate the phagosomal membrane and facilitate mycobacterial antigen translocation into the cytoplasm (Grode et al., 2005), and deletion of the urease C (ureC) gene results in an acidic phagosomal pH for optimum listeriolysin activity (Grode et al., 2005). Most importantly, VPM1002 enhanced apoptosis of infected macrophages, and the apoptotic vesicles carrying vaccine components promoted cross-priming by dendritic cells (DCs), resulting in strong $\mathrm{CD} 4^{+} \mathrm{T}$ and $\mathrm{CD} 8^{+} \mathrm{T}$ cellular immune responses (Grode et al., 2005; Winau et al., 2006) and improved stimulation of Th17 (Torchinsky et al., 2009). Macrophages infected by 
nonpathogenic species of mycobacteria trigger apoptosis, while virulent mycobacteria such as MTB and M. bovis do not efficiently induce the apoptosis (Danelishvili et al., 2003; Keane et al., 2000; Sly et al., 2003). It was demonstrated that three MTB proteins, NuoG (Velmurugan et al., 2007), SecA2 (Hinchey et al., 2007) and protein kinase E (PknE) (Jayakumar et al., 2008), had the ability to inhibit apoptosis in host cells. Furthermore, the secA2 deletion mutants of MTB triggered more macrophage apoptosis than wild-type MTB, and vaccination with a secA2-deleted strain significantly increased resistance to MTB challenge compared with standard BCG vaccination in mice and guinea pigs (Hinchey et al., 2007). In addition, a tuberculosis DNA vaccine co-expressing pro-apoptotic caspase-3 increased protection against MTB compared to DNA-encoded secreted Ag85A (Gartner et al., 2008). All of the investigations above suggest that interference with macrophage apoptosis might be a novel and practical approach to improving live TB vaccine efficacy by genetic modifications.

Apoptosis is regulated through two major pathways, the death receptor pathway and the mitochondrial pathway (Hengartner, 2000). The mitochondrial pathway is mediated by the B-cell lymphoma/leukemia 2 (Bcl-2) protein family (Green and Reed, 1998; Kelekar and Thompson, 1998; Yang and Korsmeyer, 1996). The Bcl-2 protein family was categorized as both anti-apoptotic (Bcl-2, Bcl-X $\mathrm{L}$, Bcl-w, Mcl-1, A1) and pro-apoptotic (Bax, Bak, Bok/Mtd, Bcl-Xs, Bid, Bad, Bik/Nbk, Bim, Blk) (Jacobson, 1997; Kroemer, 1997; Reed, 1997). Bax is a Bcl-2 family protein with pro-apoptotic activity, which has been shown to trigger cytochrome $c$ release from the mitochondria both in vitro and in vivo (Antonsson et al., 2001). Here, on the basis of the immune response in tuberculosis and the mitochondrial pathway of apoptosis, we hypothesized that the pro-apoptotic BAX would induce apoptosis of macrophages. Consequently, we originally 
constructed a recombinant BCG strain expressing pro-apoptotic BAX (rBCG::BAX) against Mycobacterium tuberculosis infection. The rBCG::BAX would trigger macrophage apoptosis, which would facilitate presentation of the mycobacterial antigen and confer better protective efficacy against MTB infection compared with BCG alone.

\section{Material and Methods}

\subsection{Bacterial strains and cultures}

Mycobacterium bovis BCG (Danish strain, obtained from Shanghai Biological Products Institute, China), rBCG::pMV261 and rBCG::BAX were grown at $37^{\circ} \mathrm{C}$ in Middlebrook $7 \mathrm{H} 9$ medium (Difco Laboratories, Detroit, Michigan, USA) supplemented with 0.5\% (vol./vol.) glycerol, 0.05\% (vol./vol.) Tween 80 and 10\% (vol./vol.) bovine albumin-dextrose-catalase enrichment (ADC, Becton-Dickinson and Company Sparks, MD 21152, USA) enrichment, in Sauton medium containing $0.25 \mathrm{~g}$ of $\mathrm{MgSO}_{4} \cdot 7 \mathrm{H}_{2} \mathrm{O}, 0.25 \mathrm{~g}$ of $\mathrm{K}_{2} \mathrm{HPO}_{4}, 1 \mathrm{~g}$ of citric acid, $4 \mathrm{~g}$ of sodium glutamate, $30 \mathrm{ml}$ of glycerol, $5 \mathrm{mg}$ of $\mathrm{ZnSO}_{4}$, and $25 \mathrm{mg}$ of ferrum-ammonium citrate in $500 \mathrm{ml}$ or on solid Middlebrook 7H10 medium (Difco, Becton-Dickinson and Company Sparks, MD 21152, USA) supplemented with oleic albumin-dextrose-catalase enrichment (OADC, Becton-Dickinson and Company Sparks, MD 21152, USA). Escherichia coli DH5a was grown in Luria-Bertani medium (LB, OXOID Ltd., England) at $37^{\circ} \mathrm{C}$ for cloning purposes. When required, the antibiotic kanamycin was added at a concentration of $25 \mu \mathrm{g} / \mathrm{ml}$ for Escherichia coli or recombinant BCG strain selection.

\subsection{Construction of a recombinant BCG-expressing pro-apoptotic BAX}


A recombinant Mycobacterium bovis BCG strain expressing pro-apoptotic BAX (rBCG::BAX) was constructed by transforming BCG (Danish strain) cells with a recombinant plasmid pMV261-ASP-BAX. Coding sequences for the signal peptide of Ag85B (ASP, 120bp) were amplified by PCR from Mycobacterium tuberculosis H37Rv genomic DNA using the primers ASP-Forward (5'-TATGAATTCATGACAGACGTGAGCCGAAAG-3') and ASP-Reverse (5'-ATTAAGCTTCGCGCCCGCGGTTG-3'). Coding sequences for BAX (579bp) were amplified by PCR from the cDNA of C57BL/6 mice using the primers BAX-Forward (5'-TATAAGCTTATGGACGGGTCCGGGG-3') and BAX-Reverse (5'-ATTGTCGACTCAGCCCATCTTCTTCCAGATG -3'). Both the sequences for ASP and for BAX were cloned into the Mycobacterial-E.coli shuttle vector pMV261 with an HSP60 promoter. The sequence of inserted genes was confirmed. BCG was transformed with the recombinant plasmid by electroporation as described previously (Stover et al., 1993) and selected on Middlebrook 7H10 agar containing 10\% OADC enrichment and $25 \mu \mathrm{g} / \mathrm{ml}$ kanamycin. The resulting recombinant transformants consisting of either pMV261 or pMV261-ASP-BAX were defined as rBCG::pMV261 and rBCG::BAX, respectively.

\subsection{Expression of BAX detected by Western blot}

Expression of BAX by rBCG::BAX was analyzed on SDS-PAGE and Western blot with a mouse monoclonal anti-BAX antibody (Biolegend, USA). The secondary antibody, horseradish peroxidase-conjugated goat anti-mouse immunoglobulin G (Dingguo Biotechnology, Beijing, China), was diluted $1 / 10,000$. The Western blot was illuminated in a dark room by chemiluminescence using ECL Western blotting detection reagents (Cell signaling technology, 
CST, USA) and films from KODAK (USA). The transformed BCG cells were plated on 7H10 medium with $25 \mu \mathrm{g} / \mathrm{ml}$ kanamycin added and were grown at $37^{\circ} \mathrm{C}$ for 3 weeks. Individual colonies were picked and grown in Sauton medium containing $25 \mu \mathrm{g} / \mathrm{ml}$ kanamycin. After 2 weeks, protein expression was induced by heating at $45^{\circ} \mathrm{C}$ for $60 \mathrm{~min}$. The cells were centrifuged at $12,000 \times \mathrm{g}$ for $20 \mathrm{~min}$. The culture filtrates were concentrated by vacuum-lyophilization as described previously (Bao et al., 2003). Twenty micrograms of total protein was analyzed for expression of the target protein by Western blot with a mouse monoclonal anti-BAX antibody.

\subsection{Animals and immunization}

Female C57BL/6 mice (SLAC Inc. Shanghai China), aged 6-8 weeks, were provided at a P2-level animal facility at Fudan University (Shanghai, China). Animals received free access to food and water throughout the experiment. All assays were performed in accordance with the local ethics committee. Mice were divided into four groups ( 8 per group) and were immunized subcutaneously with $1 \times 10^{6} \mathrm{CFU}$ of BCG, rBCG::pMV261 or rBCG::BAX in $100 \mu$ phosphate-buffered saline (PBS, pH 7.4). Mice were sacrificed to examine the immune responses at 4 and 12 weeks post-vaccination. The experiments were repeated twice.

\subsection{ELISPOT assays for splenocytes secreting IFN- $\gamma$ and TNF- $\alpha$}

Mice were sacrificed and the spleens were removed aseptically in RPMI-1640 medium (Gibco BRL, Invitrogen, USA) containing 10\% fetal bovine serum (FBS), $100 \mu \mathrm{g} / \mathrm{ml}$ streptomycin and $100 \mathrm{U} / \mathrm{ml}$ penicillin. Spleens were gently ground in a $70 \mu \mathrm{m}$ cell strainer, and single cell suspensions were then prepared with lympholyte-M density gradient centrifugation (Cedarlane, 
Burlington, NC, USA). Cells were counted and plated at $2.5 \times 10^{5}$ cells per well in RPMI-1640 medium. Cells were cultured for 36 hours after stimulation with Ag85B or purified protein derivative (PPD). Mouse interferon (IFN)- $\gamma$ and tumor necrosis factor (TNF)- $\alpha$ ELISPOT kits (U-CyTech Biosciences, Netherlands) were used to determine the relative number of IFN- $\gamma$ or TNF- $\alpha$ specific cells in the single cell suspensions following the manufacturer's instructions. The spot-forming units (SFU) were counted using a dissecting microscope.

\subsection{ELISA for IFN- $\gamma, T N F-\alpha, I L-2$ and IL-4 secretion from splenocytes}

To analyze the influence of rBCG::BAX on cytokine secretion, supernatants from splenocytes aseptically taken from immunized mice and stimulated ex vivo with Ag85B or PPD for 36 hours were analyzed using enzyme-linked immunosorbent assay (ELISA). All pre-coated ELISA kits were purchased from DAKEWE (Shenzheng, China). IFN- $\gamma$, TNF- $\alpha$, IL-2 and IL-4 ELISA kits were used to determine IFN- $\gamma$, TNF- $\alpha$, IL-2 and IL-4 secretion levels, respectively, in the supernatants from splenocytes according to the manufacturer's instructions.

\subsection{ELISA for antigen-specific serum antibodies}

Sera were collected from the immunized mice at 4 and 12 weeks after immunization and stored at $-80^{\circ} \mathrm{C}$. ELISA plates (Maxisorb, type 96F; Nunc, Roskilde, Denmark) were coated with $100 \mu \mathrm{l}$ Ag85B $(5 \mu \mathrm{g} / \mathrm{ml})$ per well and incubated overnight at $4^{\circ} \mathrm{C}$. The plates were washed three times with PBS containing $0.05 \%$ Tween-20 and were blocked with $200 \mu$ per well PBS containing $1 \%$ bovine serum albumin (BSA) for an hour at $37^{\circ} \mathrm{C}$ and finally washed three times as previously described. Sera diluted 1/400 were added to wells for an hour incubation followed by three wash 
steps. $100 \mu \mathrm{l}$ per well of PBS containing horseradish peroxidase-conjugated goat anti-mouse immunoglobulin G1 (IgG1, Dingguo Biotechnology, Beijing, China) and IgG2 diluted 1/10,000 were added to the wells. Plates were incubated for an hour at $37^{\circ} \mathrm{C}$, washed three times and reacted with $100 \mu \mathrm{l}$ 3,3',5,5'-tetramethyl benzidine (TMB) per well in the dark at room temperature. The reactions were stopped by adding $50 \mu \mathrm{l} 2 \mathrm{~N} \mathrm{H}_{2} \mathrm{SO}_{4}$ per well and were measured at optical density $(\mathrm{OD})=450 \mathrm{~nm}$ with an ELISA reader after $15 \mathrm{~min}$.

\subsection{Analysis by flow cytometry}

Cells were harvested and stained for surface markers on ice and analyzed by flow cytometry. All antibodies were purchased from eBioscience (USA), and the fluorescein isothiocyanate (FITC) labeled Annexin V apoptosis detection Kit I was purchased from BD (Becton-Dickinson and Company, USA). To detect macrophage apoptosis in vitro, RAW264.7 macrophages were infected with BCG, rBCG::pMV261 or rBCG::BAX in vitro for $8 \mathrm{~h}, 22 \mathrm{~h}, 47 \mathrm{~h}$ and $60 \mathrm{~h}$ and were detected by flow cytometry (FCM). To examine the potential capacity for promoting pro-apoptosis in vivo, the macrophages were isolated from C57BL/6 mice and were stained with a FITC-labeled Annexin V apoptosis detection Kit I .

With regard to evaluating the effects of $\mathrm{rBCG}: \mathrm{BAX}$ on $\mathrm{CD}^{+}{ }^{+} \mathrm{T}$ and $\mathrm{CD} 8^{+} \mathrm{T}$ lymphocytes, peripheral blood mononuclear cells (PBMC) were isolated from the peripheral blood of immunized mice at different time points and were stained with a combination of FITC-conjugated anti-mouse CD4 and PE-conjugated anti-mouse CD8a, followed by detection with FCM.

To reveal the mechanism by which $\mathrm{rBCG}: \mathrm{BAX}$ enhances Th1 protective immune responses, mice 
were sacrificed and the macrophages were harvested. For each mouse, macrophages were stained with a combination of FITC-conjugated anti-mouse CD14 and PE-Cyane5-conjugated anti-mouse CD11b for 20 min on ice. Next, macrophages were divided into four samples and were stained with phycoerythrin (PE)-conjugated anti-mouse MHC-I, PE-conjugated anti-mouse MHC-II, PE-conjugated anti-mouse CD80(B7-1), and PE-conjugated anti-mouse CD86(B7-2) for 20 min on ice, respectively. Finally, the samples were measured by FCM.

\subsection{Statistical analysis}

Prism 5.0 software (GraphPad) was used for all statistical analyses. The data are expressed as the means \pm standard deviation (SD), and a one-way ANOVA was used to analyze the differences with a Turkey post-hoc test. $\mathrm{P}$ values of less than $0.05(*)$ and $\mathrm{P}$ values of less than $0.01(* *)$ were considered statistically significant. All results were representative of at least two independent experiments.

\section{Theory}

Mycobacterium tuberculosis, the etiologic agent of tuberculosis, is an intracellular pathogen known for evading mechanisms of the protective immune response and causing chronic disease in human (Briken and Miller, 2008). Despite accumulating evidence, the molecular mechanisms underlying MTB infection-induced cell death remain enigmatic. Traditionally, MTB has been thought to survive and replicate in macrophages. Currently, investigations have illustrated that MTB-infected resident alveolar macrophages and other types of cells are recruited to the lungs in response to inflammatory signals: neutrophils, monocytes, interstitial macrophages, and dendritic 
cells (DCs) (Srivastava et al., 2014). Because MTB invades the host through the lung alveoli, it has been widely believed that alveolar macrophages are the first phagocytes to encounter MTB and are the predominant resident cells of TB (Srivastava et al., 2014).

Although humans have developed more complicated immune systems to detect and eradicate persisting intracellular pathogens, MTB has evolved several strategies to adapt to macrophages by developing anti-apoptosis mechanisms and concomitantly reducing exposure to apoptosis-inducing molecules on its cell surface (Briken et al., 2004; Karakousis et al., 2004). In the early stages of infection, the dissemination of MTB from alveolar macrophages is likely delayed as a consequence of the ability of MTB to inhibit apoptosis of the infected host cells (Blomgran et al., 2012; Keane et al., 2000; Velmurugan et al., 2007). Evidence has demonstrated that alveolar macrophages participate with DCs in cellular responses even though they do not directly elicit T-cell responses (Blomgran et al., 2012). Therefore, the long delay in initiation of T-cell immunity after MTB infection in mice (Chackerian et al., 2002) is partly due to the ability of the pathogen to reside in alveolar macrophages and to inhibit apoptosis of infected host cells before releasing to DCs, which terminally migrate to local lymph nodes and initiate T-cell responses (Srivastava et al., 2014).

Generally speaking, apoptosis can be induced through two pathways: the extrinsic pathway, which is triggered by death receptors such as Fas/CD95 or TNF- $\alpha$ receptor 1, which activate Caspase-8/10, and the intrinsic pathway, which is elicited upon intracellular stress monitored by the mitochondria and initiated by activation of Caspase-9 (Maiuri et al., 2007; Siegel, 2006). Both pathways converge at the activation of Caspase-3/6/7, which subsequently triggers apoptosis 
(Maiuri et al., 2007; Siegel, 2006). The release of cytochrome $c$ from the mitochondria has been shown to play an essential role in many apoptotic signaling cascades through the activation of downstream effector caspases. Further studies demonstrated that BAX is a trigger of cytochrome $c$ release (Antonsson et al., 2001).

Importantly, virulent mycobacteria inhibit the apoptosis of macrophages in order to evade the host immune system, whereas macrophages undergo apoptosis after infection with avirulent mycobacteria (Danelishvili et al., 2003; Keane et al., 2000; Sly et al., 2003). Several investigations have illustrated that virulent MTB is capable of inhibiting the intrinsic pathway of apoptosis through the regulation of pro-apoptotic and anti-apoptotic proteins. An in vitro investigation has indicated that MTB can induce and inhibit apoptosis by regulating the expression of Bax and Bcl-2, respectively, indicating that the fate of infected cells relies on pro-apoptotic and anti-apoptotic signals (Mogga et al., 2002). In addition, some studies have demonstrated that the anti-apoptotic protein Bcl-w is up-regulated in cells infected by the MTB H37Rv strain but not the H37Ra strain (Spira et al., 2003), whereas the pro-apoptotic protein Bad is inactivated during MTB H37Rv infection (Maiti et al., 2001). When the bacillary load exceeds approximately 20 per macrophage, virulent MTB leads to cell death via lysosomal permeabilization, releasing hydrolases that promote Bax/Bak-independent mitochondrial damage and necrosis (Lee et al., 2011). Furthermore, macrophages infected with the attenuated MTB strain H37Ra induced apoptosis, which suppressed bacterial replication and facilitated antigen presentation (Danelishvili et al., 2003; Keane et al., 2000; Sly et al., 2003). In contrast, in macrophages infected with the virulent strain $\mathrm{H} 37 \mathrm{Rv}$, the amino-terminal domain of annexin-1 was removed by proteolysis, subsequently inhibiting completion of the apoptotic envelope, which led to necrosis of infected 
macrophages (Gan et al., 2008). Virulent MTB therefore escapes the host defense system by blocking formation of the apoptotic envelope, which results in macrophage necrosis and dissemination of pathogen in the lung (Gan et al., 2008). Thus, virulent MTB is eventually transformed into the extracellular persistent pathogen that characterizes advanced pulmonary tuberculosis (Lee et al., 2011). In line with these results, investigations demonstrated that the apoptosis of macrophages causes the release of apoptotic vesicles carrying the pathogens to uninfected DCs, which were indispensable for subsequent cross-presentation of mycobacterial protein and glycolipid to T cells through MHC-I and CD1b (Schaible et al., 2003). Therefore, the pro-apoptotic response is beneficial to the host because MTB bacteria need to reside and persist in macrophages.

\section{Results}

\subsection{Construction of a recombinant BCG strain expressing pro-apoptotic BAX}

To construct a novel recombinant BCG strain capable of inducing apoptosis of infected macrophages, the gene Bax was inserted into the mycobacterial-E.coli shuttle vector pMV261. The sequences encoding the signal peptide of secreted mycolyl transferase antigen 85B (ASP) of Mycobacterium tuberculosis were also inserted into vector pMV261 to allow secretory expression of BAX. The recombinant clones (containing plasmid pMV261 and recombinant plasmid pMV261-ASP-BAX) were designated rBCG::pMV261 and rBCG::BAX, respectively. As a result of the sequence of the signal peptide of $\mathrm{Ag} 85 \mathrm{~B}$, the expression of the target protein could be detected in the culture filtrates of $\mathrm{rBCG}: \mathrm{BAX}$ by Western blot with mouse monoclonal antibodies recognizing BAX. The Western-blot analysis revealed BAX at the expected molecular weight 
from the culture filtrates of $\mathrm{rBCG}: \mathrm{BAX}$, whereas BAX was absent from that of BCG or rBCG::pMV261 (Fig. 1).

\subsection{Essential role of the rBCG::BAX in promoting macrophage apoptosis}

The ability of MTB to evade the host immune response is due to its ability to inhibit macrophage apoptosis, which is typically triggered upon infection of phagocytic cells by vacuolar bacterial pathogens (Menaker and Jones, 2003; Navarre and Zychlinsky, 2000; Yrlid and Wick, 2000). In vitro studies indicated that the death of infected cells by apoptosis contributes to the extermination of intracellular pathogens through the activation of as-yet-undefined bactericidal mechanisms (Riendeau and Kornfeld, 2003; Sly et al., 2003). Apoptosis, or programmed cell death, is characterized by a loss of membrane phospholipid asymmetry, leading to the exposure of phosphatidylserine at the surface of the cells (Koopman et al., 1994). In this study, we detected apoptotic cells by flow cytometry (FCM) using the binding of fluorescein isothiocyanate (FITC)-labeled annexin V to phosphatidylserine. RAW264.7 macrophages were infected with BCG, rBCG::pMV261 or rBCG::BAX in vitro for $8 \mathrm{~h}, 22 \mathrm{~h}, 47 \mathrm{~h}$ and $60 \mathrm{~h}$ and were detected by flow cytometry (FCM). The results demonstrated that a small amount of macrophage apoptosis occurred after infection with BCG, rBCG::pMV261 or rBCG::BAX in vitro for $8 \mathrm{~h}, 22 \mathrm{~h}$, and $47 \mathrm{~h}$ (data not shown), whereas almost all of the macrophages underwent apoptosis after infection for 60 h. rBCG::BAX significantly increased the apoptotic cells, in contrast to BCG, in vitro (Fig. 2A and 2B). Macrophages were isolated from C57BL/6 mice immunized with BCG, rBCG::pMV261 or $\mathrm{rBCG}: \mathrm{BAX}$ for 12 weeks, and the apoptotic cells were examined by FCM. The results also confirmed that $\mathrm{rBCG}:: \mathrm{BAX}$ had a significant ability to induce apoptosis when compared with 
BCG. (Fig. $2 \mathrm{C}$ and 2D)

\subsection{Evaluation of IFN- $\gamma, T N F-\alpha, I L-2$ and $I L-4$ secretion}

Single splenocyte suspension from immunized mice were harvested and tested for interferon (IFN)- $\gamma$ and tumor necrosis factor (TNF)- $\alpha$ at $36 \mathrm{~h}$ post-stimulation with Ag85B or MTB purified protein derivative (PPD). An enzyme-linked immunospot assay (ELISPOT) was used to enumerate the relative numbers of IFN- $\gamma$ or TNF- $\alpha$ secreting cells in single cell suspension from the spleens of C57BL/6 mice immunized with BCG, rBCG::pMV261 or rBCG::BAX for 12 weeks. The results showed that $\mathrm{rBCG}: \mathrm{BAX}$ significantly increased the IFN- $\gamma$ spot-forming units (SFU) in response to Ag85B, whereas IFN- $\gamma$ SFU responses to PPD were not significant. (Fig. 3 A) The TNF- $\alpha$ SFU responses to Ag85B and PPD were not significant in comparison with BCG. (Fig. 3 B)

To analyze the effects of $\mathrm{rBCG}:$ BAX on cytokine secretion, supernatants of splenocytes taken from immunized mice and stimulated ex vivo with Ag85B and PPD for 36 hours were analyzed using enzyme-linked immunosorbent assay (ELISA). In response to Ag85B, IFN- $\gamma$ release from splenocytes derived from C57BL/6 mice immunized with rBCG::BAX was significantly elevated compared with splenocytes from mice given BCG, but IFN- $\gamma$ release was not significant in responses to PPD (Fig. 3 C). A similar result was obtained for TNF- $\alpha$ release in response to Ag85B and PPD (Fig. 3 D). In response to Ag85B, interleukin (IL)-2 release from splenocytes isolated from C57BL/6 mice immunized with rBCG::BAX was significantly increased in comparison with splenocytes from mice given BCG, whereas IL-2 release was not significant in response to PPD (Fig. 3 E). Notably, in response to Ag85B, IL-4 release from splenocytes derived 
from C57BL/6 mice immunized with $\mathrm{BBCG}: \mathrm{BAX}$ was significantly decreased compared with splenocytes from mice given BCG, while IL-4 release was not significant in response to PPD (Fig. 3 F). All of the analyses on cytokine secretion showed that rBCG::BAX markedly enhanced Th1 immune responses.

\subsection{Evaluation of $C D 4^{+} \mathrm{T}$ and $\mathrm{CD}^{+} \mathrm{T}$ lymphocytes derived from $\mathrm{PBMC}$}

To evaluate the influence of $\mathrm{rBCG}: \mathrm{BAX}$ on $\mathrm{CD} 4^{+} \mathrm{T}$ and $\mathrm{CD} 8^{+} \mathrm{T}$ lymphocytes, peripheral blood mononuclear cells (PBMC) were isolated from the peripheral blood of mice immunized with BCG, rBCG::pMV261 or rBCG::BAX and were detected by means of FCM at different time points. The ratios of $\mathrm{CD} 4^{+} \mathrm{T} / \mathrm{CD} 8^{+} \mathrm{T}$ demonstrated that $\mathrm{rBCG}$ : $\mathrm{BAX}$ was superior to $\mathrm{BCG}$ with respect to the maintenance of a persistent ratio of $\mathrm{CD}^{+}{ }^{+} \mathrm{T} / \mathrm{CD} 8^{+} \mathrm{T}$ in peripheral blood during the whole investigation period (Fig. 4 A).

\subsection{Evaluation of Ag85B-specific serum antibody responses}

To determine the induction of Th1/Th2 immune responses in animals, mice were sacrificed to examine Ag85B-specific serum antibody levels of $\operatorname{IgG} 1$ and $\operatorname{IgG} 2 \mathrm{~b}$ by ELISA at various time points after immunization with BCG, rBCG::pMV261 or rBCG::BAX. The ratio of IgG2b/IgG1 from mice immunized with $\mathrm{rBCG}$ ::BAX was markedly higher than that of mice immunized with BCG for 12 weeks (Fig. 4 B). The results revealed that rBCG::BAX was superior to BCG for the induction of Th1 protective immune responses.

\subsection{Expression of MHC and co-stimulatory molecules on macrophages}


To explore the mechanism by which $\mathrm{BBCG}: \mathrm{BAX}$ enhances Th1 protective immune responses, the expression levels of major histocompatibility complex (MHC) and co-stimulatory molecules on macrophages isolated from mice immunized with BCG, rBCG::pMV261 or rBCG::BAX were examined by FCM. The results illustrated that $\mathrm{rBCG}: \mathrm{BAX}$ significantly up-regulated the expression levels of MHC-II (Fig. 5A, 5B, 5C and 5D) and B7.1 (CD80) co-stimulatory molecules (Fig. 5 E, F, G and H) on macrophages, whereas those of MHC-I (data not shown) and B7.2 (CD86) were not significant (data not shown). The up-regulated expression of MHC-II and B7.1 (CD80) co-stimulatory molecules could facilitate the activation of $\mathrm{T}$ cells and presumably promote antigen presentation, thereby enhancing Th1 protective immune responses against MTB.

\section{Discussion}

In this study, a rBCG::BAX strain was originally constructed from a parental BCG (Danish stain) with a hypothesis that $\mathrm{rBCG}: \mathrm{BAX}$ would promote the apoptosis of macrophages. The Western-blot analysis revealed that the expression of the target protein BAX could be detected in the culture filtrates of $\mathrm{rBCG}:$ BAX. The results (data not shown) of sera anti-BAX antibody response were consistent with the consequence of Western-blot analysis. Sera were collected from the immunized mice at 4 weeks after immunization and were determined anti-BAX antibody by ELISA. The data illustrated the level of antibody response in the sera of mice from different groups against recombinant purified protein BAX. Since the target gene Bax did not exist in parental BCG, there were no obvious IgG titers detected in the sera of mice immunized with PBS, BCG or rBCG::pMV261. However, IgG antibody titers as high as 1:6400 were obtained in the sera of mice immunized with $\mathrm{rBCG}: \mathrm{BAX}$, which were significantly higher than those in the sera 
of mice immunized with of BCG. The results revealed that $\mathrm{rBCG}:$ :BAX expressed the BAX protein in vivo, and induced the antibody response.

In the process of cellular immunity against MTB, macrophages also serve as antigen-presenting cells, in which the mycobacterial antigens are degraded into immunogenic polypeptides and presented to $\mathrm{T}$ cell through MHC to initiate adaptive immunity (Xu et al., 2014). The results confirmed that $\mathrm{rBCG}:$ BAX significantly increased the apoptosis of infected macrophages both in vitro and in vivo, which would facilitate antigen presentation. Both T-cell populations and macrophages act synergistically in regulating the immune system to eliminate MTB. The CD4 ${ }^{+}$ T-helper (Th) cells polarize into different subsets (Mosmann and Sad, 1996). Th1 immune responses, which are indispensable for the defense against intracellular pathogens such as MTB, produce IL-2 for T-cell activation, IFN- $\gamma$, or TNF- $\alpha$ for macrophage activation (Cooper, 2009; Khader et al., 2007; Korn et al., 2009). Th2 cells and regulatory T cells (Treg) counteract Th1-mediated protection via IL-4, transforming growth factor (TGF), or IL-10 (Belkaid and Tarbell, 2009). In our investigation, rBCG::BAX significantly increased Ag85B-specific IFN- $\gamma$ enzyme-linked immunospot responses, IFN- $\gamma$ secretion, and IL-2 secretion, while it significantly decreased Ag85B-specific IL-4. Somewhat unexpectedly, the differences were not statistically significant with regard to cytokine (TNF- $\alpha$, IFN- $\gamma$, IL-2 and IL-4) secretion in response to PPD stimulation. Additionally, CD8+T cells also produce IFN- $\gamma$ and TNF, which activate macrophages (Cooper, 2009; Kaufmann, 2006). They also function as cytolytic T lymphocytes (CTL) by secreting perforin and granulysin, which lyse host cells and directly attack MTB (Stenger et al., 1998). 
Furthermore, antibody isotype titers are often detected to evaluate the polarity of the immune response. The gene for IgG2a is deleted in C57BL/6 (Martin and Lew, 1998). The IgG2b isotype is associated with Th1-mediated protective immune responses, while the IgG1 isotype links to Th2-mediated pathological immune responses. In our study, rBCG::BAX markedly increased the ratio of Ag85B-specific IgG2b/IgG1, which suggested a shift towards Th1 immune responses. The results are consistent with the increase in IFN- $\gamma$ and IL-2 secretion and the decrease in IL-4 production.

To construct $\mathrm{rBCG}: \mathrm{BAX}$ by electroporation, both the sequences for signal peptide of Ag85B (ASP, 120bp) and for BAX (579bp) were cloned into the Mycobacterial-E.coli shuttle vector pMV261 to get a recombinant plasmid pMV261-ASP-BAX. The major role of ASP is to allow secretory expression of target protein BAX. In our experiments, the data demonstrated that rBCG::BAX could express the target protein BAX, enhanced the apoptosis of macrophages infected with it and subsequently facilitated mycobacterial antigen presentation by macrophages through up-regulating the expression of the MHC-II and CD80 co-stimulation molecules. As a result of the property of $\mathrm{rBCG}: \mathrm{BAX}$, it enhanced Th1 immune responses. Therefore, the enhanced immune responses reported in the manuscript would be induced by the pro-apoptotic protein BAX.

Activation of T-cells is mediated through two essential signals provided by activated macrophages that play an indispensable role in initiating the Th-1 protective immune response against MTB (de la Barrera et al., 2004). Because most effector $\mathrm{T}$ cells are in the lung interstitium, alveolar macrophages, which reside in the airspaces of the lungs, have limited contact with effector $\mathrm{T}$ cells 
(Srivastava et al., 2014). Nevertheless, in the lungs of MTB-infected mice, alveolar macrophages are different from recruited interstitial macrophages in their up-regulated expression of CD11c, MHC class II, and co-stimulatory molecules such as CD80 and CD86 (Gonzalez-Juarrero et al., 2003; Wolf et al., 2007), indicating that alveolar macrophages should be able to be recognized by effector T cells (Srivastava et al., 2014). T-cells recognize the antigens on MHC-I or MHC-II for activation to regulate the functions of macrophages, causing either inhibition or promotion of intracellular MTB growth. MTB can interfere with the functions of phagosomes (Frehel and Rastogi, 1987; Rastogi et al., 1992) and subsequently reduce the capacity of mycobacterial antigens to be processed and presented via the MHC class-II molecules (Pancholi et al., 1993). It is well known that expression of co-stimulatory molecules on macrophages serves as a second signal and is essential for activation and proliferation of antigen specific T-cells (de la Barrera et al., 2004). The down-regulation of these co-stimulatory molecules on macrophages can be one of the virulence-associated mechanisms in MTB infection. In our study, the results illustrated that rBCG::BAX significantly up-regulated the expression of MHC-II and B7.1 (CD80) co-stimulatory molecules on macrophages, suggesting that $\mathrm{rBCG}: \mathrm{BAX}$ may potentially facilitate antigen presentation. It was reported that B7.1 was responsible for the development of Th-1-like cells and that B7.2 (CD86) enhances the expansion of Th2 lymphocytes (Brown et al., 1996; Freeman et al., 1995; Keane-Myers et al., 1998; Kuchroo et al., 1995; Racke et al., 1995; Ranger et al., 1996). Moreover, interaction of co-stimulatory molecules B7.1 and B7.2 on antigen-presenting cells with CD28 on T cells is essential for optimal activation of antigen recognizing T-cells. In agreement with our results, a current investigation demonstrated that Wnt/beta-catenin signaling possesses the potential to promote macrophage apoptosis in response to mycobacterial infection (Wu et al., 
2014). Notably, the activation of Wnt/beta-catenin signaling was able to increase apoptosis in BCG-infected mouse macrophage-like Raw 264.7 cells in part by a mitochondria-dependent apoptosis pathway (Wu et al., 2014). Furthermore, immunoblotting analysis indicated that Wnt/beta-catenin signaling induced cell apoptosis partly through a caspase-dependent apoptosis pathway by down-regulation of the anti-apoptotic protein Mcl-1 and up-regulation of pro-apoptotic proteins Bax and cleaved-caspase-3, as well as by enhancement of caspase-3 activity in BCG-infected RAW264.7 cells (Wu et al., 2014). Therefore, the expression of co-stimulatory molecules that in turn indicate the activated state of the immune response is indispensable for promoting the expansion of specific T-cells, especially the protective Th- 1 type cells during tuberculosis infection.

\section{Conclusions}

Conventionally, the induction of $\mathrm{CD} 4^{+} \mathrm{T}$ helper type 1 (Th1) cells in mice is protective, while the induction of Th2 cells can increase susceptibility to MTB (Cooper et al., 1993; Flynn et al., 1993) In our study, we constructed a novel recombinant BCG stain (rBCG::BAX) against Mycobacterium tuberculosis infection. The immunogenicity of the rBCG::BAX strain was superior to BCG in terms of the potential to induce the Th1 protective immune responses, presumably because it could enhance the apoptosis of macrophages infected with rBCG::BAX and subsequently facilitated mycobacterial antigen presentation by macrophages through up-regulating the expression of the MHC-II and CD80 co-stimulation molecules. Therefore, rBCG::BAX might be a potential tuberculosis vaccine candidate for further study.

\section{Conflict of interest}


The authors declare that there are no potential conflicts of interest.

\section{Acknowledgements}

This work was supported by grants from the National Major Special Projects (2012ZX10003008-001), the NSF of China (31100660) and the NSF of Shanghai Sci. Tech. Committee (11ZR1401600).

\section{Glossary}

BCG, bacille Calmette Guérin; rBCG::BAX, a novel recombinant BCG strain expressing pro-apoptotic BAX; VPM1002, rBCG $\Delta \mathrm{UreC}:$ Hly; MTB, Mycobacterium tuberculosis; TB, tuberculosis; Ag85, antigen 85; ASP, signal peptide of Ag85B; PPD, MTB purified protein derivative; ADC, bovine albumin-dextrose-catalase enrichment; IFN- $\gamma$, interferon-gamma; TNF- $\alpha$, tumor necrosis factor-alpha; IL, interleukin; DCs, dendritic cells; ELISPOT, an enzyme-linked immunospot assay; ELISA, enzyme-linked immunosorbent assay; MHC, major histocompatibility complex; FCM, flow cytometry; FITC, fluorescein isothiocyanate; Th1, CD4+ T helper type 1, CTL, cytolytic T lymphocytes. 


\section{References:}

(2013) WHO publishes Global tuberculosis report 2013. Euro Surveill 18.

Andersen P. and Kaufmann S. H. (2014) Novel vaccination strategies against tuberculosis. Cold Spring Harb Perspect Med 4.

Antonsson B., Montessuit S., Sanchez B. and Martinou J. C. (2001) Bax is present as a high molecular weight oligomer/complex in the mitochondrial membrane of apoptotic cells. J Biol Chem 276, 11615-23.

Bao L., Chen W., Zhang H. and Wang X. (2003) Virulence, immunogenicity, and protective efficacy of two recombinant Mycobacterium bovis bacillus Calmette-Guerin strains expressing the antigen ESAT-6 from Mycobacterium tuberculosis. Infect Immun 71, 1656-61.

Belkaid Y. and Tarbell K. (2009) Regulatory T cells in the control of host-microorganism interactions (*). Annu Rev Immunol 27, 551-89.

Blomgran R., Desvignes L., Briken V. and Ernst J. D. (2012) Mycobacterium tuberculosis inhibits neutrophil apoptosis, leading to delayed activation of naive CD4 T cells. Cell Host Microbe 11 , 81-90.

Briken V. and Miller J. L. (2008) Living on the edge: inhibition of host cell apoptosis by Mycobacterium tuberculosis. Future Microbiol 3, 415-22.

Briken V., Porcelli S. A., Besra G. S. and Kremer L. (2004) Mycobacterial lipoarabinomannan and related lipoglycans: from biogenesis to modulation of the immune response. Mol Microbiol 53, 391-403.

Brown J. A., Titus R. G., Nabavi N. and Glimcher L. H. (1996) Blockade of CD86 ameliorates Leishmania major infection by down-regulating the Th2 response. J Infect Dis 174, 1303-8.

Chackerian A. A., Alt J. M., Perera T. V., Dascher C. C. and Behar S. M. (2002) Dissemination of Mycobacterium tuberculosis is influenced by host factors and precedes the initiation of T-cell immunity. Infect Immun 70, 4501-9.

Colditz G. A., Brewer T. F., Berkey C. S., Wilson M. E., Burdick E., Fineberg H. V. and Mosteller F. (1994) Efficacy of BCG vaccine in the prevention of tuberculosis. Meta-analysis of the published literature. JAMA 271, 698-702.

Cooper A. M. (2009) Cell-mediated immune responses in tuberculosis. Annu Rev Immunol 27, 393-422.

Cooper A. M., Dalton D. K., Stewart T. A., Griffin J. P., Russell D. G. and Orme I. M. (1993) Disseminated tuberculosis in interferon gamma gene-disrupted mice. J Exp Med 178, 2243-7.

Danelishvili L., McGarvey J., Li Y. J. and Bermudez L. E. (2003) Mycobacterium tuberculosis infection causes different levels of apoptosis and necrosis in human macrophages and alveolar epithelial cells. Cell Microbiol 5, 649-60

de la Barrera S., Aleman M., Musella R., Schierloh P., Pasquinelli V., Garcia V., Abbate E. and Sasiain M. C. (2004) IL-10 down-regulates costimulatory molecules on Mycobacterium tuberculosis-pulsed macrophages and impairs the lytic activity of CD4 and CD8 CTL in tuberculosis patients. Clin Exp Immunol 138, 128-38.

Flynn J. L., Chan J., Triebold K. J., Dalton D. K., Stewart T. A. and Bloom B. R. (1993) An essential role for interferon gamma in resistance to Mycobacterium tuberculosis infection. J Exp Med 178, 2249-54.

Freeman G. J., Boussiotis V. A., Anumanthan A., Bernstein G. M., Ke X. Y., Rennert P. D., Gray G. S., 
Gribben J. G. and Nadler L. M. (1995) B7-1 and B7-2 do not deliver identical costimulatory signals, since B7-2 but not B7-1 preferentially costimulates the initial production of IL-4. Immunity 2, 523-32.

Frehel C. and Rastogi N. (1987) Mycobacterium leprae surface components intervene in the early phagosome-lysosome fusion inhibition event. Infect Immun 55, 2916-21.

Gan H., Lee J., Ren F., Chen M., Kornfeld H. and Remold H. G. (2008) Mycobacterium tuberculosis blocks crosslinking of annexin-1 and apoptotic envelope formation on infected macrophages to maintain virulence. Nat Immunol 9, 1189-97.

Gartner T., Romano M., Suin V., Kalai M., Korf H., De Baetselier P. and Huygen K. (2008) Immunogenicity and protective efficacy of a tuberculosis DNA vaccine co-expressing pro-apoptotic caspase-3. Vaccine 26, 1458-70.

Gonzalez-Juarrero M., Shim T. S., Kipnis A., Junqueira-Kipnis A. P. and Orme I. M. (2003) Dynamics of macrophage cell populations during murine pulmonary tuberculosis. J Immunol 171, 3128-35.

Green D. R. and Reed J. C. (1998) Mitochondria and apoptosis. Science 281, 1309-12.

Grode L., Ganoza C. A., Brohm C., Weiner J. R., Eisele B. and Kaufmann S. H. (2013) Safety and immunogenicity of the recombinant BCG vaccine VPM1002 in a phase 1 open-label randomized clinical trial. Vaccine 31, 1340-8.

Grode L., Seiler P., Baumann S., Hess J., Brinkmann V., Nasser E. A., Mann P., Goosmann C., Bandermann S., Smith D., Bancroft G. J., Reyrat J. M., van Soolingen D., Raupach B. and Kaufmann S. H. (2005) Increased vaccine efficacy against tuberculosis of recombinant Mycobacterium bovis bacille Calmette-Guerin mutants that secrete listeriolysin. J Clin Invest $\mathbf{1 1 5}$, 2472-9.

Hatherill M. (2011) Prospects for elimination of childhood tuberculosis: the role of new vaccines. Arch Dis Child 96, 851-6.

Hengartner M. O. (2000) The biochemistry of apoptosis. Nature 407, 770-6.

Hinchey J., Lee S., Jeon B. Y., Basaraba R. J., Venkataswamy M. M., Chen B., Chan J., Braunstein M., Orme I. M., Derrick S. C., Morris S. L., Jacobs W. J. and Porcelli S. A. (2007) Enhanced priming of adaptive immunity by a proapoptotic mutant of Mycobacterium tuberculosis. J Clin Invest $\mathbf{1 1 7}$, 2279-88.

Jacobson M. D. (1997) Apoptosis: Bcl-2-related proteins get connected. Curr Biol 7, R277-81.

Jayakumar D., Jacobs W. J. and Narayanan S. (2008) Protein kinase E of Mycobacterium tuberculosis has a role in the nitric oxide stress response and apoptosis in a human macrophage model of infection. Cell Microbiol 10, 365-74.

Karakousis P. C., Bishai W. R. and Dorman S. E. (2004) Mycobacterium tuberculosis cell envelope lipids and the host immune response. Cell Microbiol 6, 105-16.

Kaufmann S. H. (2006) Envisioning future strategies for vaccination against tuberculosis. Nat Rev Immunol 6, 699-704.

Kaufmann S. H. and Gengenbacher M. (2012) Recombinant live vaccine candidates against tuberculosis. Curr Opin Biotechnol 23, 900-7.

Kaufmann S. H., Cotton M. F., Eisele B., Gengenbacher M., Grode L., Hesseling A. C. and Walzl G. (2014) The BCG replacement vaccine VPM1002: from drawing board to clinical trial. Expert Rev Vaccines 13, 619-30.

Keane J., Remold H. G. and Kornfeld H. (2000) Virulent Mycobacterium tuberculosis strains evade apoptosis of infected alveolar macrophages. J Immunol 164, 2016-20. 
Keane-Myers A. M., Gause W. C., Finkelman F. D., Xhou X. D. and Wills-Karp M. (1998) Development of murine allergic asthma is dependent upon B7-2 costimulation. J Immunol 160, 1036-43.

Kelekar A. and Thompson C. B. (1998) Bcl-2-family proteins: the role of the BH3 domain in apoptosis. Trends Cell Biol 8, 324-30.

Khader S. A., Bell G. K., Pearl J. E., Fountain J. J., Rangel-Moreno J., Cilley G. E., Shen F., Eaton S. M., Gaffen S. L., Swain S. L., Locksley R. M., Haynes L., Randall T. D. and Cooper A. M. (2007) IL-23 and IL-17 in the establishment of protective pulmonary CD4+ T cell responses after vaccination and during Mycobacterium tuberculosis challenge. Nat Immunol 8, 369-77.

Koopman G., Reutelingsperger C. P., Kuijten G. A., Keehnen R. M., Pals S. T. and van Oers M. H. (1994) Annexin V for flow cytometric detection of phosphatidylserine expression on B cells undergoing apoptosis. Blood 84, 1415-20.

Korn T., Bettelli E., Oukka M. and Kuchroo V. K. (2009) IL-17 and Th17 Cells. Annu Rev Immunol 27, 485-517.

Kroemer G. (1997) The proto-oncogene Bcl-2 and its role in regulating apoptosis. Nat Med 3, 614-20.

Kuchroo V. K., Das M. P., Brown J. A., Ranger A. M., Zamvil S. S., Sobel R. A., Weiner H. L., Nabavi N. and Glimcher L. H. (1995) B7-1 and B7-2 costimulatory molecules activate differentially the Th1/Th2 developmental pathways: application to autoimmune disease therapy. Cell 80, 707-18

Lee J., Repasy T., Papavinasasundaram K., Sassetti C. and Kornfeld H. (2011) Mycobacterium tuberculosis induces an atypical cell death mode to escape from infected macrophages. PLoS One 6 , e18367.

Maiti D., Bhattacharyya A. and Basu J. (2001) Lipoarabinomannan from Mycobacterium tuberculosis promotes macrophage survival by phosphorylating Bad through a phosphatidylinositol 3-kinase/Akt pathway. J Biol Chem 276, 329-33.

Maiuri M. C., Zalckvar E., Kimchi A. and Kroemer G. (2007) Self-eating and self-killing: crosstalk between autophagy and apoptosis. Nat Rev Mol Cell Biol 8, 741-52.

Martin R. M. and Lew A. M. (1998) Is IgG2a a good Th1 marker in mice? Immunol Today 19, 49.

Menaker R. J. and Jones N. L. (2003) Fascination with bacteria-triggered cell death: the significance of Fas-mediated apoptosis during bacterial infection in vivo. Microbes Infect 5, 1149-58.

Mogga S. J., Mustafa T., Sviland L. and Nilsen R. (2002) Increased Bcl-2 and reduced Bax expression in infected macrophages in slowly progressive primary murine Mycobacterium tuberculosis infection. Scand J Immunol 56, 383-91.

Mosmann T. R. and Sad S. (1996) The expanding universe of T-cell subsets: Th1, Th2 and more. Immunol Today $17,138-46$.

Navarre W. W. and Zychlinsky A. (2000) Pathogen-induced apoptosis of macrophages: a common end for different pathogenic strategies. Cell Microbiol 2, 265-73

Pancholi P., Mirza A., Bhardwaj N. and Steinman R. M. (1993) Sequestration from immune CD4+ T cells of mycobacteria growing in human macrophages. Science 260, 984-6.

Racke M. K., Scott D. E., Quigley L., Gray G. S., Abe R., June C. H. and Perrin P. J. (1995) Distinct roles for B7-1 (CD-80) and B7-2 (CD-86) in the initiation of experimental allergic encephalomyelitis. J Clin Invest 96, 2195-203.

Ranger A. M., Das M. P., Kuchroo V. K. and Glimcher L. H. (1996) B7-2 (CD86) is essential for the development of IL-4-producing T cells. Int Immunol 8, 1549-60. 
Rastogi N., Bachelet M. and Carvalho D. S. J. (1992) Intracellular growth of Mycobacterium avium in human macrophages is linked to the increased synthesis of prostaglandin E2 and inhibition of the phagosome-lysosome fusions. FEMS Microbiol Immunol 4, 273-9.

Reed J. C. (1997) Double identity for proteins of the Bcl-2 family. Nature 387, 773-6.

Riendeau C. J. and Kornfeld H. (2003) THP-1 cell apoptosis in response to Mycobacterial infection. Infect Immun 71, 254-9.

Schaible U. E., Winau F., Sieling P. A., Fischer K., Collins H. L., Hagens K., Modlin R. L., Brinkmann V. and Kaufmann S. H. (2003) Apoptosis facilitates antigen presentation to T lymphocytes through MHC-I and CD1 in tuberculosis. Nat Med 9, 1039-46.

Siegel R. M. (2006) Caspases at the crossroads of immune-cell life and death. Nat Rev Immunol 6, 308-17.

Sly L. M., Hingley-Wilson S. M., Reiner N. E. and McMaster W. R. (2003) Survival of Mycobacterium tuberculosis in host macrophages involves resistance to apoptosis dependent upon induction of antiapoptotic Bcl-2 family member Mcl-1. J Immunol 170, 430-7.

Spira A., Carroll J. D., Liu G., Aziz Z., Shah V., Kornfeld H. and Keane J. (2003) Apoptosis genes in human alveolar macrophages infected with virulent or attenuated Mycobacterium tuberculosis: a pivotal role for tumor necrosis factor. Am J Respir Cell Mol Biol 29, 545-51.

Srivastava S., Ernst J. D. and Desvignes L. (2014) Beyond macrophages: the diversity of mononuclear cells in tuberculosis. Immunol Rev 262, 179-92.

Stenger S., Hanson D. A., Teitelbaum R., Dewan P., Niazi K. R., Froelich C. J., Ganz T., Thoma-Uszynski S., Melian A., Bogdan C., Porcelli S. A., Bloom B. R., Krensky A. M. and Modlin R. L. (1998) An antimicrobial activity of cytolytic T cells mediated by granulysin. Science $282,121-5$

Stover C. K., Bansal G. P., Hanson M. S., Burlein J. E., Palaszynski S. R., Young J. F., Koenig S., Young D. B., Sadziene A. and Barbour A. G. (1993) Protective immunity elicited by recombinant bacille Calmette-Guerin (BCG) expressing outer surface protein A (OspA) lipoprotein: a candidate Lyme disease vaccine. J Exp Med 178, 197-209.

Svenson S., Kallenius G., Pawlowski A. and Hamasur B. (2010) Towards new tuberculosis vaccines. Hum Vaccin 6, 309-17.

Torchinsky M. B., Garaude J., Martin A. P. and Blander J. M. (2009) Innate immune recognition of infected apoptotic cells directs T(H) 17 cell differentiation. Nature 458, 78-82.

Trunz B. B., Fine P. and Dye C. (2006) Effect of BCG vaccination on childhood tuberculous meningitis and miliary tuberculosis worldwide: a meta-analysis and assessment of cost-effectiveness. Lancet 367, 1173-80.

Velmurugan K., Chen B., Miller J. L., Azogue S., Gurses S., Hsu T., Glickman M., Jacobs W. J., Porcelli S. A. and Briken V. (2007) Mycobacterium tuberculosis nuoG is a virulence gene that inhibits apoptosis of infected host cells. PLoS Pathog 3, e110.

Winau F., Weber S., Sad S., de Diego J., Hoops S. L., Breiden B., Sandhoff K., Brinkmann V., Kaufmann S. H. and Schaible U. E. (2006) Apoptotic vesicles crossprime CD8 T cells and protect against tuberculosis. Immunity 24, 105-17.

Wolf A. J., Linas B., Trevejo-Nunez G. J., Kincaid E., Tamura T., Takatsu K. and Ernst J. D. (2007) Mycobacterium tuberculosis infects dendritic cells with high frequency and impairs their function in vivo. J Immunol 179, 2509-19.

Wu X., Deng G., Hao X., Li Y., Zeng J., Ma C., He Y., Liu X. and Wang Y. (2014) A 
caspase-dependent pathway is involved in Wnt/beta-catenin signaling promoted apoptosis in Bacillus Calmette-Guerin infected RAW264.7 macrophages. Int J Mol Sci 15, 5045-62.

Xu G., Wang J., Gao G. F. and Liu C. H. (2014) Insights into battles between Mycobacterium tuberculosis and macrophages. Protein Cell 5, 728-36.

Yang E. and Korsmeyer S. J. (1996) Molecular thanatopsis: a discourse on the BCL2 family and cell death. Blood 88, 386-401.

Yrlid U. and Wick M. J. (2000) Salmonella-induced apoptosis of infected macrophages results in presentation of a bacteria-encoded antigen after uptake by bystander dendritic cells. J Exp Med 191, 613-24.

Zumla A., George A., Sharma V., Herbert R. H., Oxley A. and Oliver M. (2015) The WHO 2014 Global tuberculosis report-further to go. Lancet Glob Health 3, e10-2. 


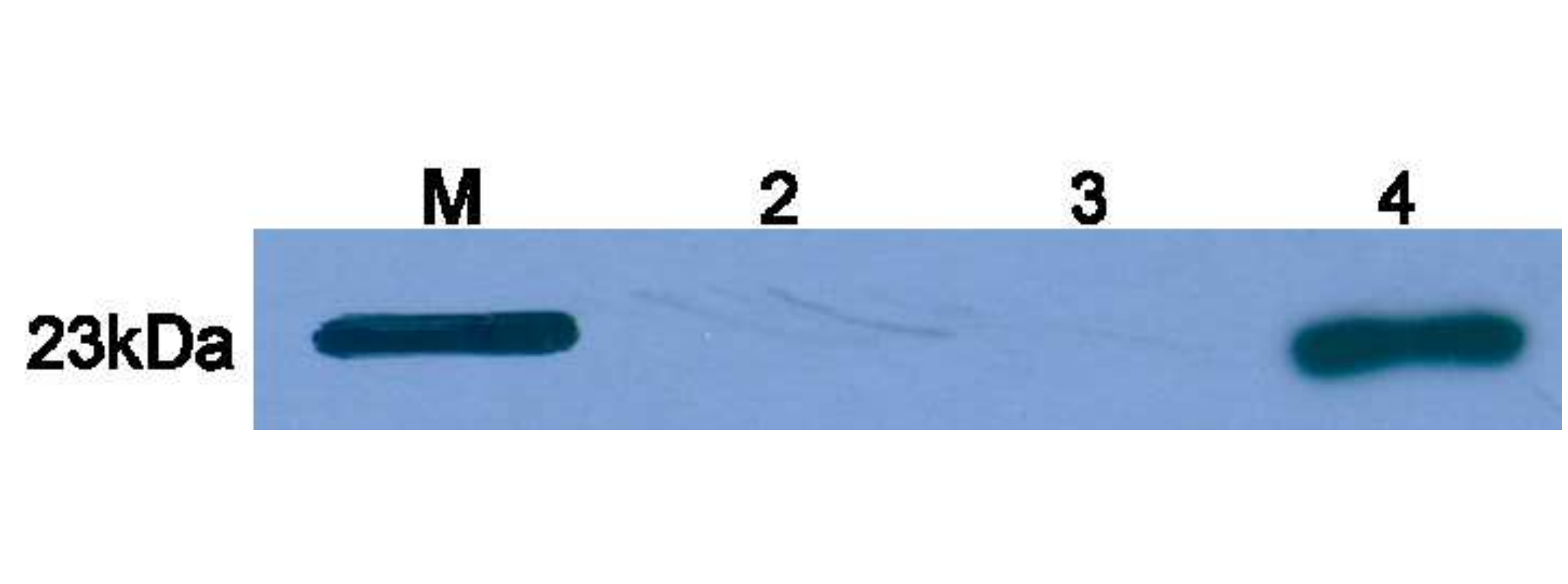
(

M

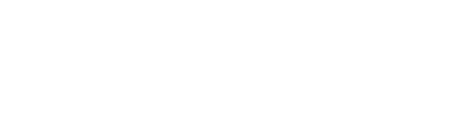

$2-1$
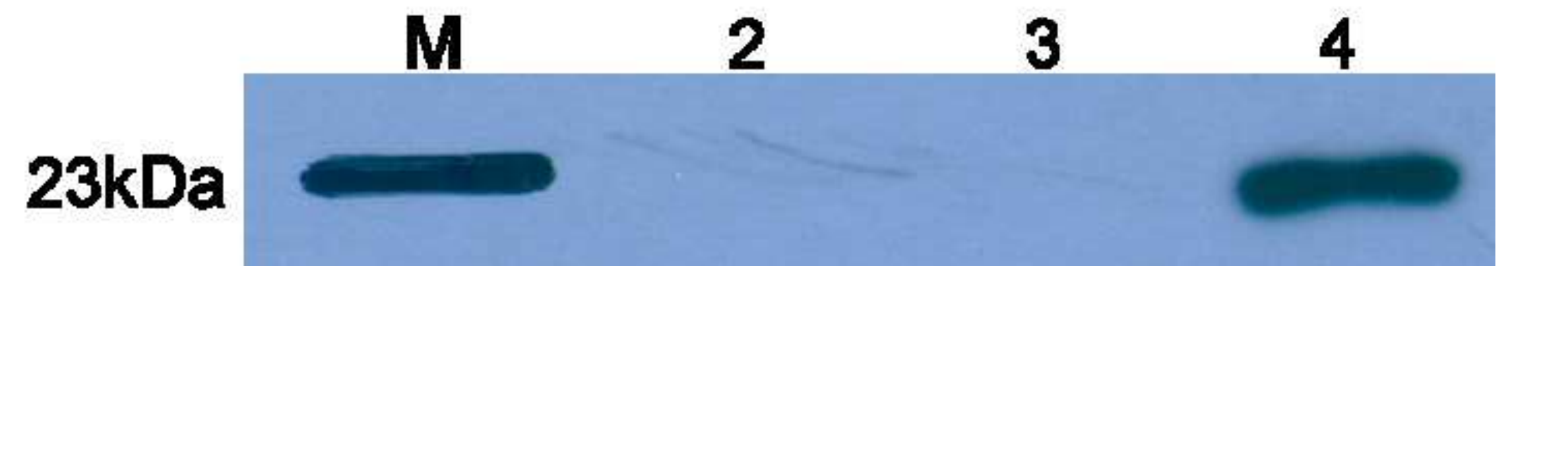

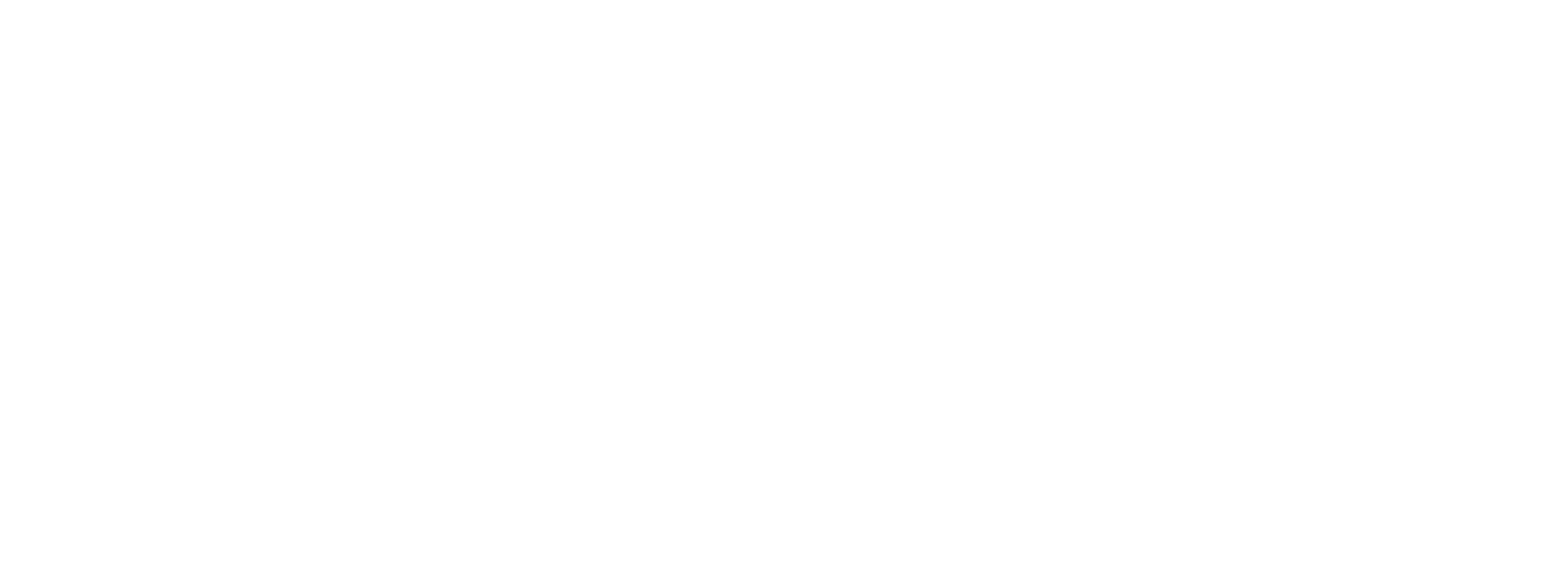
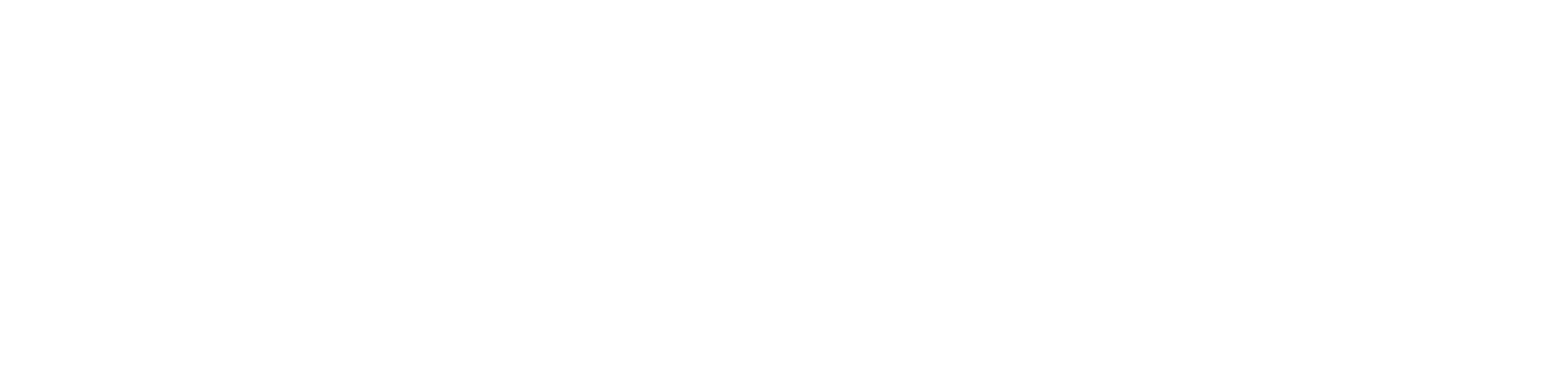

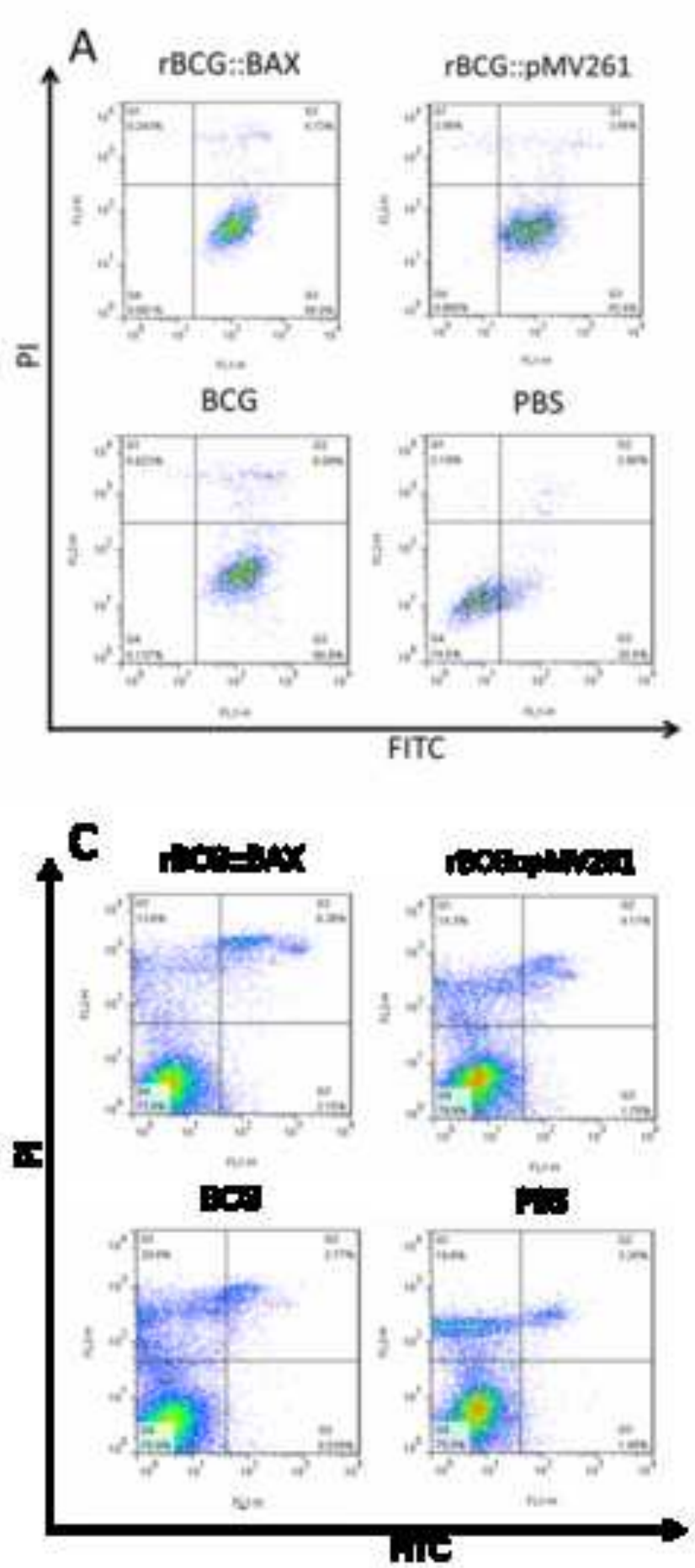

B
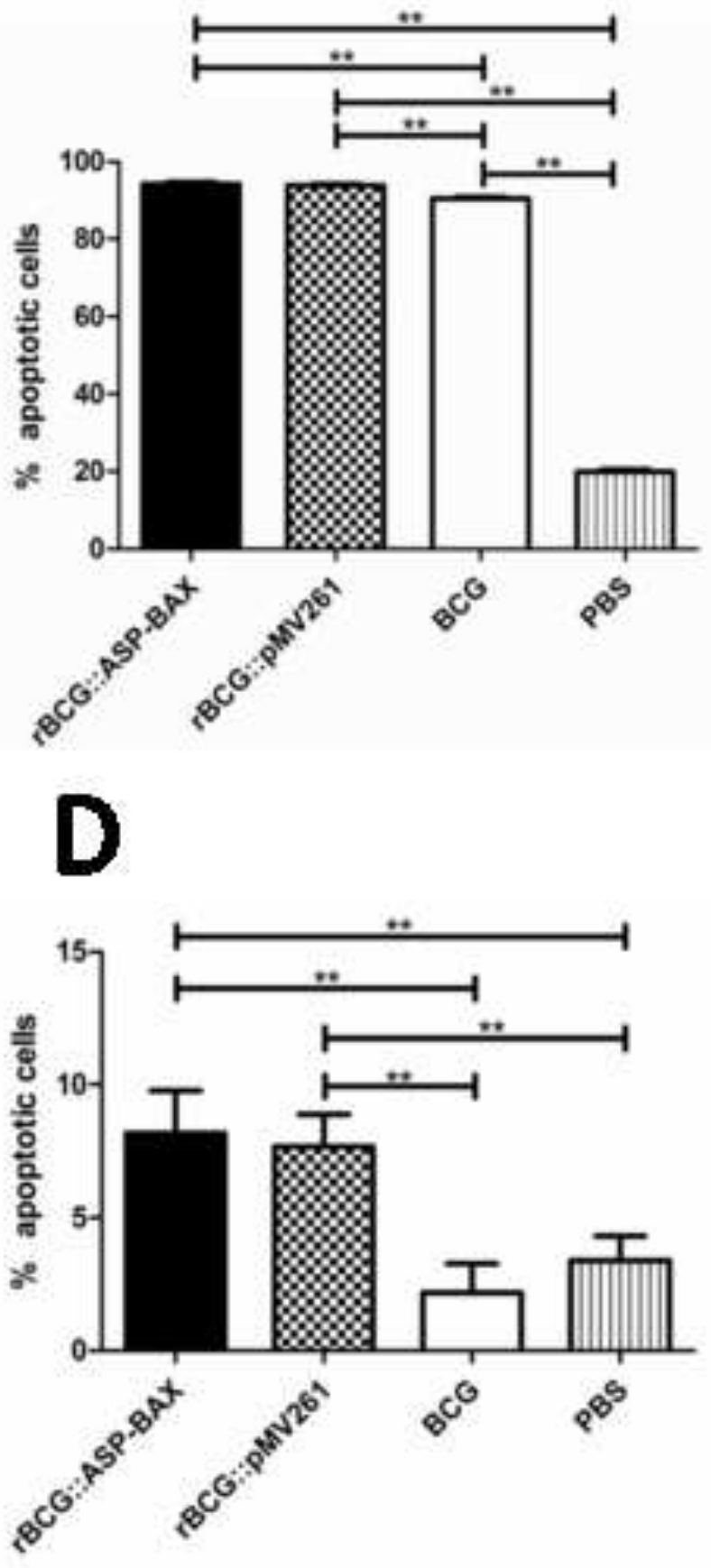
A

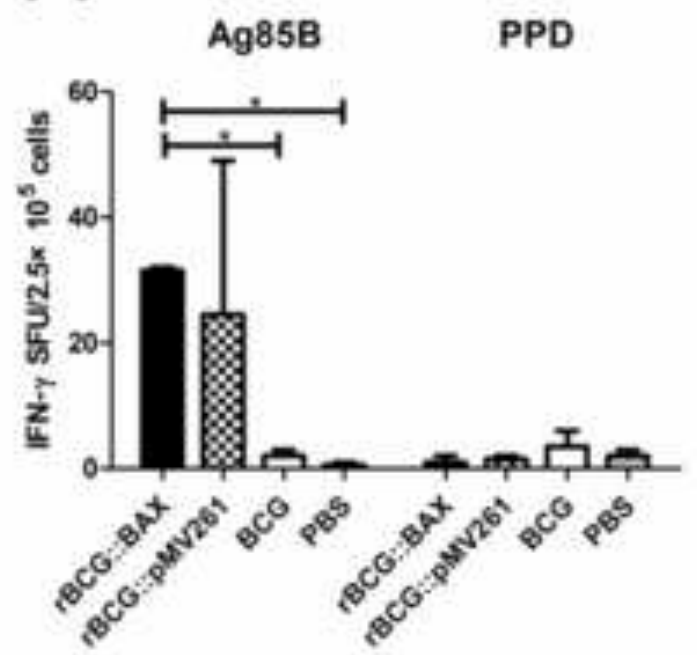

C

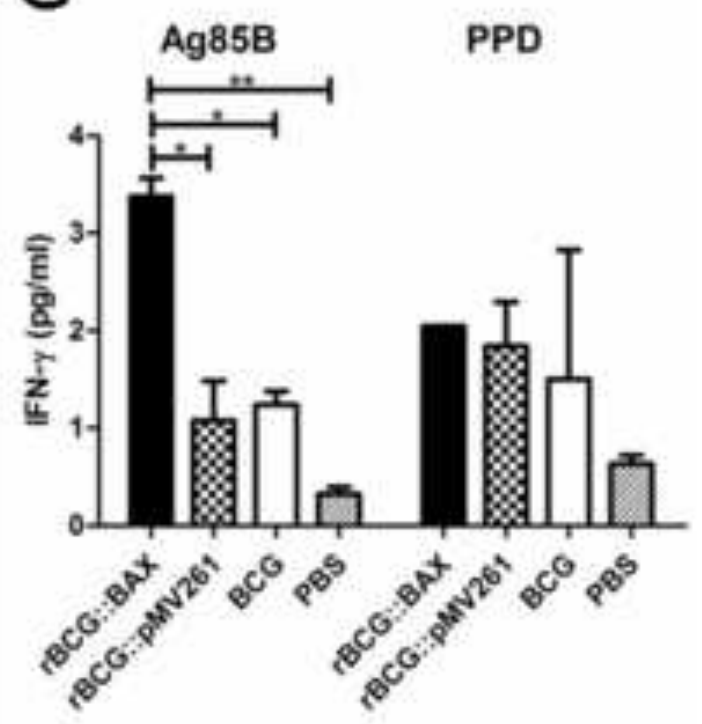

E

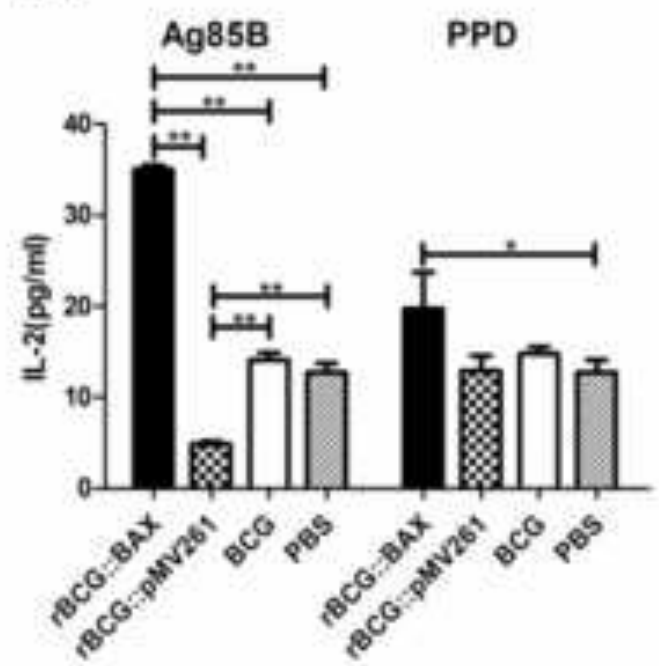

B

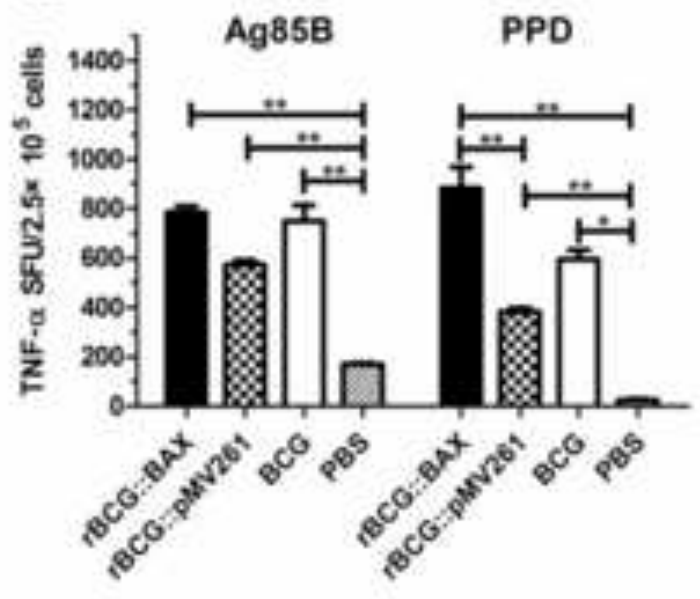

D

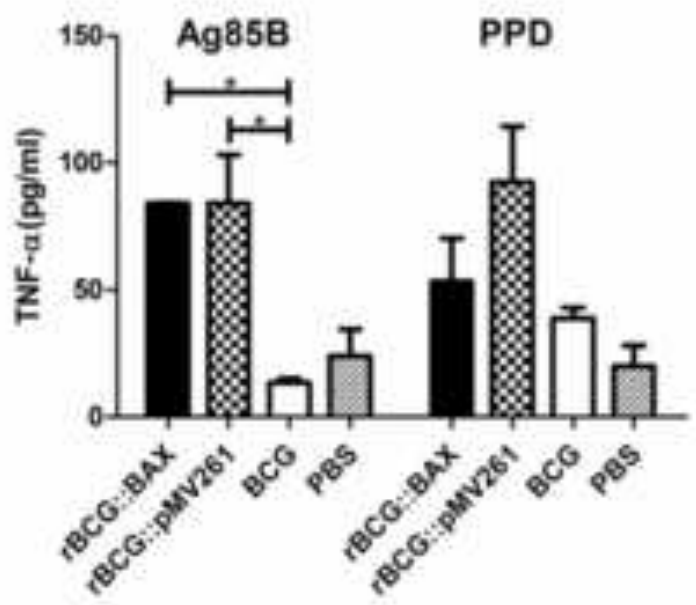

F

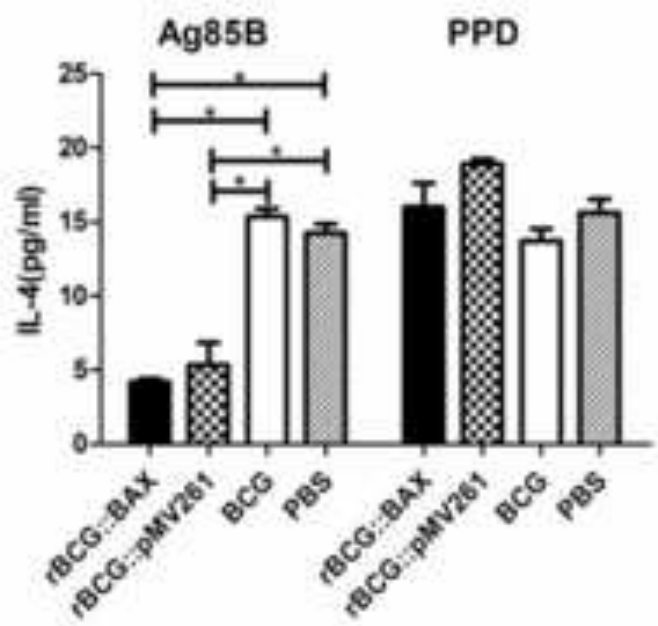


A

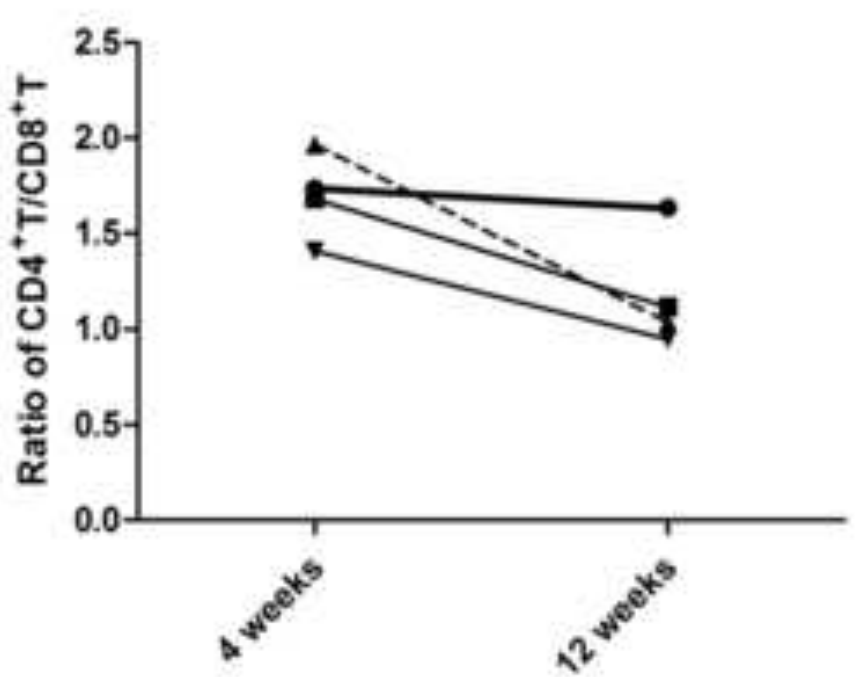

B

Anti-Ag85B lgG2b/lgG1

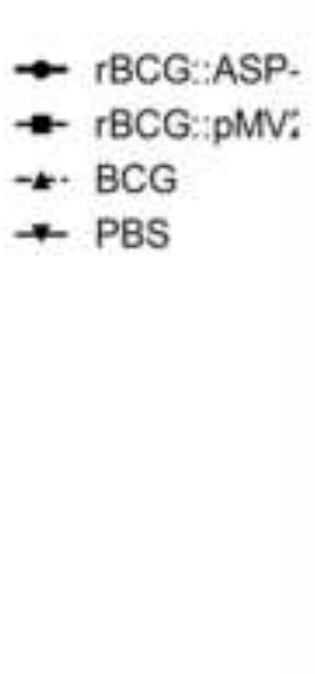

- rBCG:BAX

- rBCG: $p M N 261$

-* BCG

$\rightarrow$ PBS

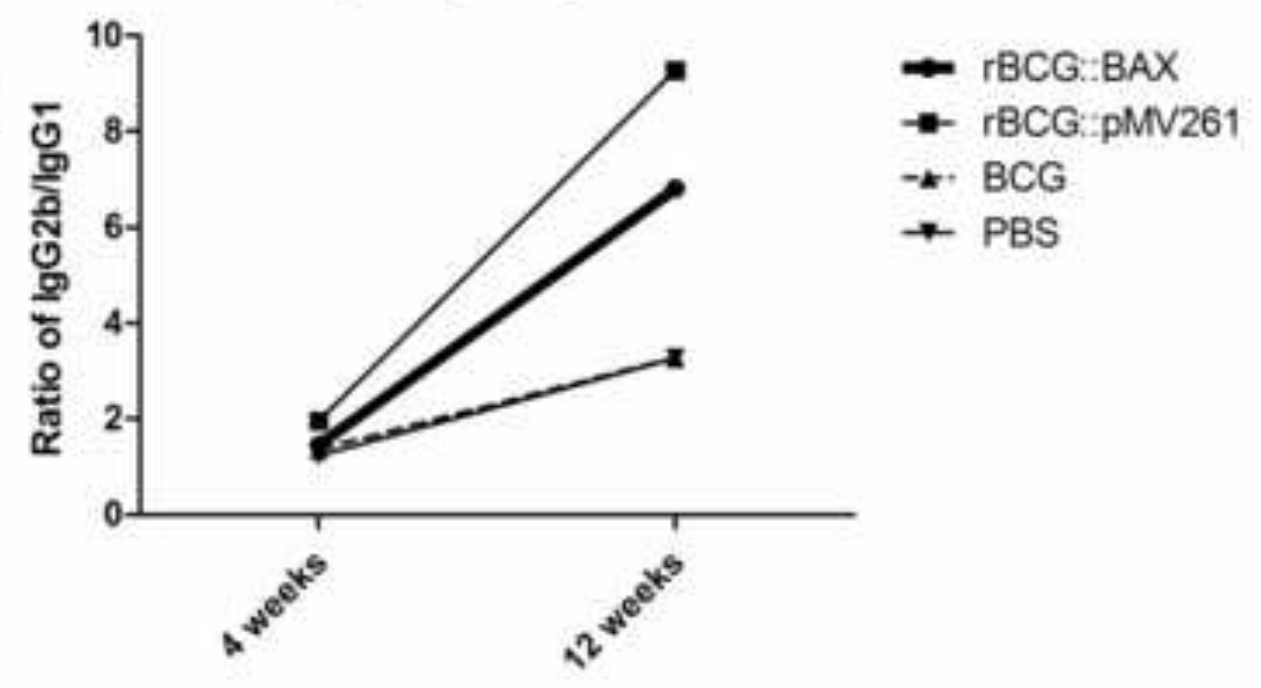



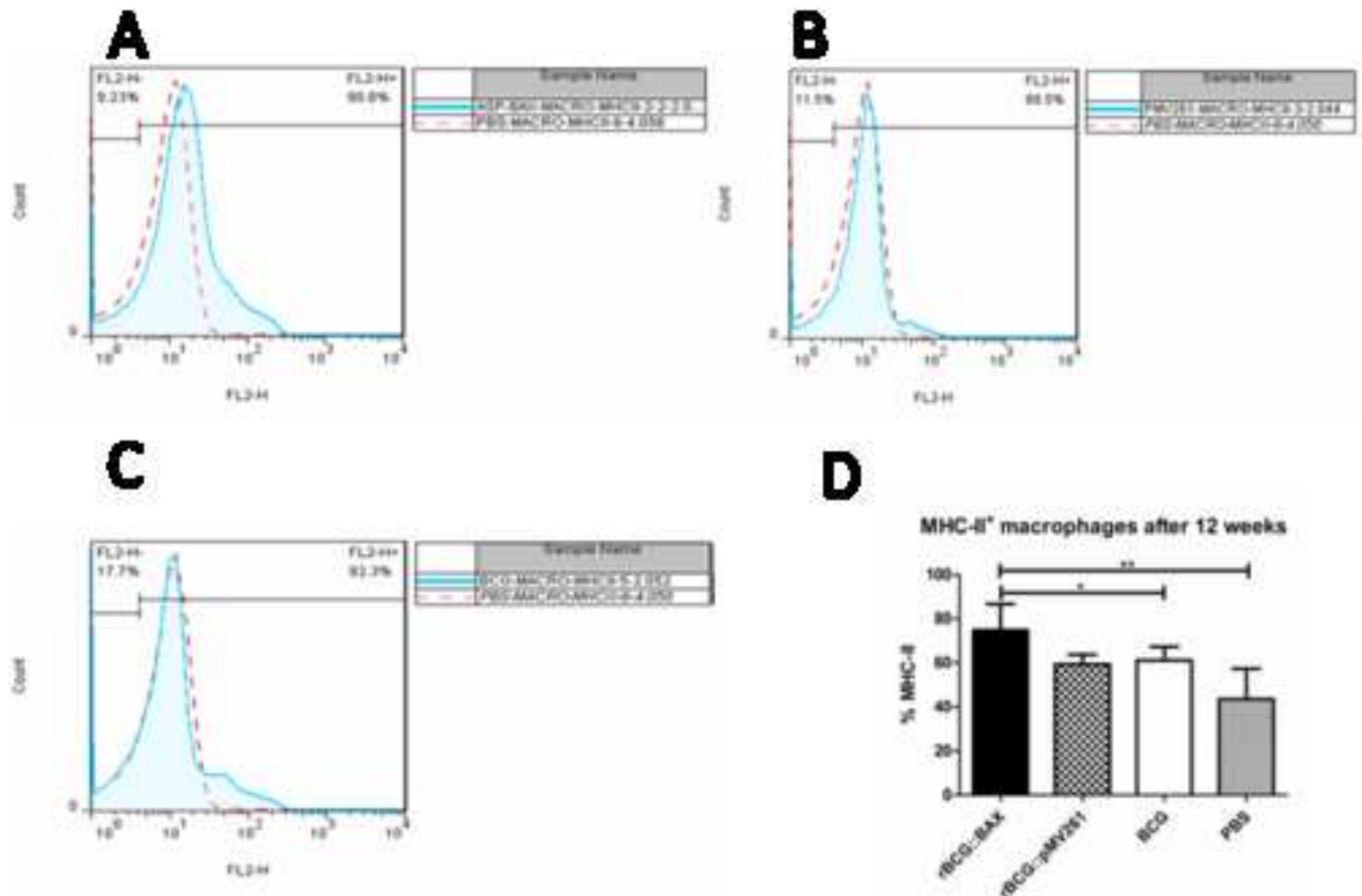

MHC-I" macrophages after 12 weeks
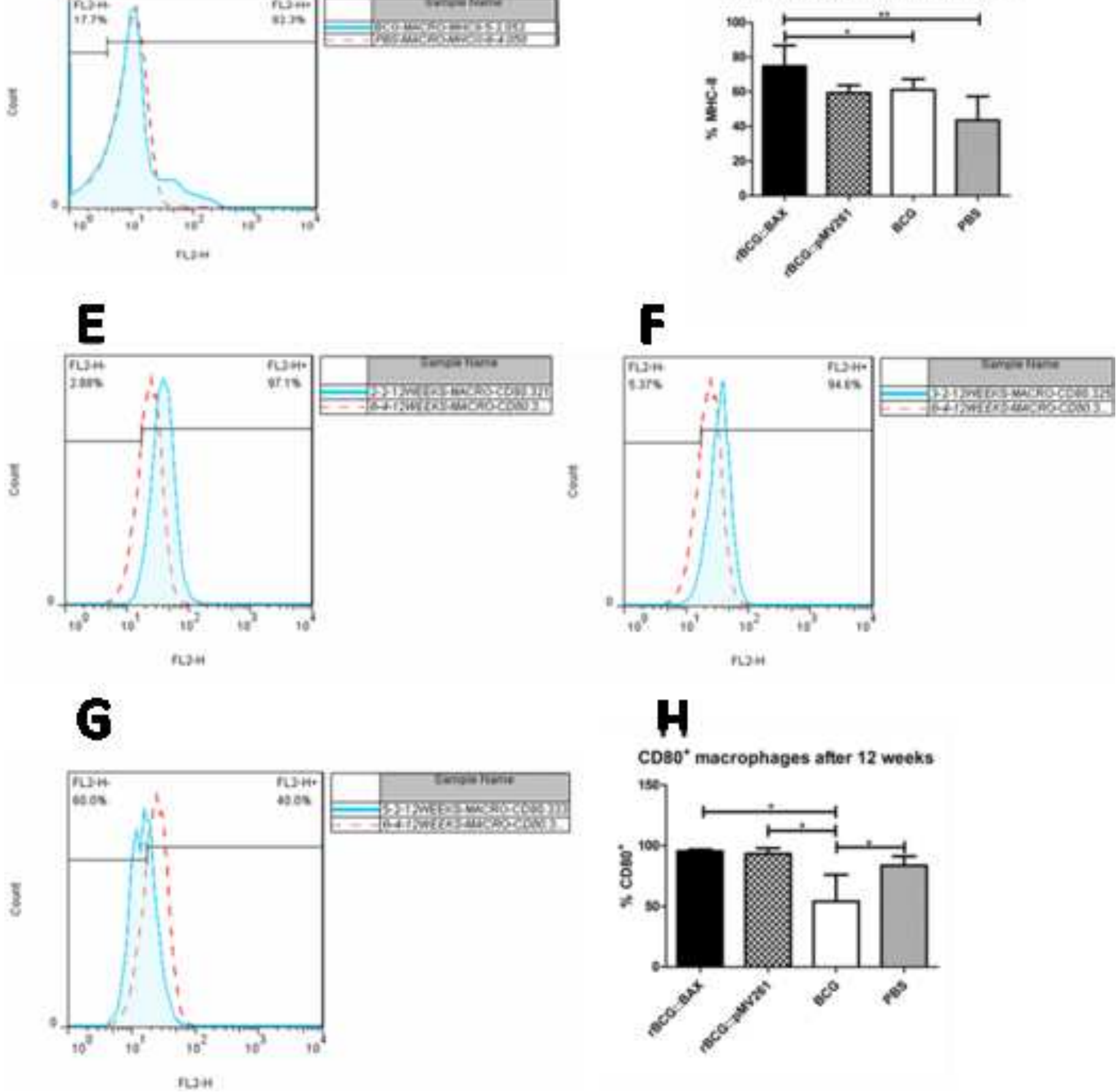


\section{Figure Captions}

2 Fig. 1 Western-blot analysis. The culture filtrates were analyzed for expression of BAX by

3 Western-blot with mouse monoclonal anti-BAX antibody (Biolegend, USA). Lane 1. Marker; Lane 2. BCG; Lane 3. rBCG::pMV261; Lane 4. rBCG::BAX

Fig. 2 Induction of apoptosis in macrophages infected with various stains in vitro and in vivo.

Mouse macrophage-like Raw 264.7 cells were stimulated with PBS, BCG, rBCG::pMV261 or rBCG::BAX in vitro for $60 \mathrm{~h}$ and were detected by flow cytometry (FCM) (Fig. 2A, Fig. 2B). The macrophages were isolated from C57BL/6 mice and tested by FCM after immunization for 12 weeks (Fig. 2C, Fig. 2D). The data are expressed as the means \pm standard deviation (SD). $* \mathrm{P}<$ $0.05,{ }^{* *} \mathrm{P}<0.01$ by one-way ANOVA with a Tukey post-hoc test.

Fig. 3 The cytokine secretion of spleen cells isolated from C57BL/6 mice was analyzed 12 weeks after immunization with PBS, BCG, rBCG::pMV261 or rBCG::BAX. The isolated spleen cells were plated in duplicate at $2.5 \times 10^{5}$ cells per well and co-cultured with Ag85B (10 $\mu \mathrm{g} / \mathrm{ml}$ ) or purified protein derivative (PPD, $10 \mu \mathrm{g} / \mathrm{ml}$ )) for $36 \mathrm{~h}$ at $37^{\circ} \mathrm{C}$ and $5 \% \mathrm{CO}_{2}$. The ELISPOT kit (U-CyTech Biosciences, Netherlands) was used to determine the relative number of IFN- $\gamma$ (Fig. 3 A) or TNF- $\alpha$ (Fig. 3B)-specific cells in the single cell suspensions according to the manufacturer's instructions. The production of IFN- $\gamma$ (Fig. 3C), TNF- $\alpha$ (Fig. 3D), IL-2 (Fig. 3E), and IL-4 (Fig. 3 F) in the supernatants of splenocytes was detected by ELISA (DAKEWE, Shenzheng, China) after stimulation with Ag85B and PPD for 36 hours ex vivo. The data are expressed as the means \pm standard deviation (SD). Statistical analyses were performed by one-way ANOVA with a Turkey post-hoc test. $* \mathrm{P}<0.05$, $* * \mathrm{P}<0.01$ 
Fig. 4 The effects on $\mathrm{CD4}^{+} \mathrm{T}$ and $\mathrm{CD8}^{+} \mathrm{T}$ lymphocytes of PBMC were analyzed by $\mathrm{FCM}$. The peripheral blood mononuclear cells (PBMC) were isolated from the peripheral blood of C57BL/6 mice 4 weeks and 12 weeks after immunization and were detected by FCM (Fig. 4A). The persistent ratio of $\mathrm{CD} 4^{+} \mathrm{T} / \mathrm{CD} 8^{+} \mathrm{T}$ is beneficial for maintenance of normal immunity against Mycobacterium tuberculosis. The serum antibody response was analyzed by ELISA in C57BL/6 (Fig. 4B). Sera were collected from mice immunized with PBS, BCG, rBCG::pMV261, or rBCG::BAX at various time points (4 weeks, 12 weeks) and examined for IgG1 and IgG2b against $\mathrm{Ag} 85 \mathrm{~B}$ by ELISA. The IgG2b isotype is relevant to Th1-mediated protective immune responses, whereas the IgG1 isotype is linked to Th2-mediated immune responses. Increases in the ratio of IgG2b/IgG1 suggest a switch to Th1-mediated immune responses. The data are expressed as the means \pm standard deviation (SD).

Fig. 5 Expression of MHC and co-stimulatory molecules on macrophages was detected by FCM. Macrophages isolated from mice immunized with PBS, BCG, rBCG::pMV261 or rBCG::BAX for 12 weeks were examined by FCM for expression of MHC-I (data not shown), MHC-II (Fig. 5 A,B,C and D), CD80 (Fig. 5 E, F, G and H) and CD86 (data not shown). The data are expressed as the means \pm standard deviation (SD). Statistical analyses were performed by one-way ANOVA with a Turkey post-hoc test. $* \mathrm{P}<0.05$, $* * \mathrm{P}<0.01$. 